\title{
Colombia: entre el rechazo y la negociación. Análisis de las campañas electorales sobre la negociación con los grupos guerrilleros en las elecciones presidenciales de 2018
}

\author{
Colombia: between rejection and negotiation. An analysis of the \\ electoral campaigns concerning the negotiation with the guerrilla \\ groups during the Presidential elections of 2018
}

\author{
Alexandra Jima ${ }^{1}$ Miguel Paradela-López ${ }^{2}$ Darío Pineda ${ }^{3}$ Ana María Serrano ${ }^{4}$ Liliana Marcela Arango ${ }^{5}$ \\ Sara Liliana Areiza ${ }^{6}$ César Augusto García ${ }^{7}$ Katherin Loaiza ${ }^{8}$ María Isabel Orozco ${ }^{9}$ Juan Esteban Parra ${ }^{10}$ David Zapata ${ }^{11}$
}

\begin{abstract}
Resumen
El presente artículo realiza un análisis de las agendas presidenciales de Colombia en las elecciones a la Presidencia de la República de 2018, enfatizando en las principales propuestas de los candidatos, en relación con la implementación de lo acordado en La Habana con la insurgencia de las Fuerzas Armadas Revolucionarias de Colombia - Ejército del Pueblo (FARC-EP) y el proceso de negociación con el Ejército de Liberación Nacional (ELN). Para ello se realiza un seguimiento a los programas políticos de los diferentes candidatos a la Presidencia, sus comparecencias públicas y los mensajes emitidos a través de sus redes sociales. En este sentido, el artículo muestra cómo durante la campaña electoral se configuraron dos posturas: una basada en rectificar, ajustar o modificar el acuerdo suscrito con las FARC-EP y priorizar la respuesta armada al ELN; y otra sustentada en el mantenimiento de los Acuerdos de La Habana y la búsqueda de una solución negociada al conflicto con el ELN. Se concluye, mostrando cómo la campaña electoral a la Presidencia de la República de 2018 supuso un agudizamiento de la polarización y radicalización en la resolución de los conflictos internos.
\end{abstract}

Palabras claves: Elecciones presidenciales; discursos presidenciales; Colombia; Acuerdos de La Habana; FARC-EP; ELN.

Abstract

This article aims at analyzing several candidates' agenda during the first and second rounds of the 2018 Presidential elections in Colombia. This analysis focuses on the candidates' proposals concerning the implementation of La Habana Peace Agreement with the Fuerzas Armadas Revolucionarias de Colombia -Ejército del Pueblo (FARC-EP) and the negotiation with the Ejército de Liberación Nacional (ELN). With that purpose in mind, this article evaluates political statements, public appearances, and social media communication from all the candidates to the presidency. As a result, this research evidences two different points: one aimed at rectifying, adjusting, or modifying the agreement with FARC-EP and prioritizing an armed response against the ELN; and other aimed at maintaining the La Habana Peace Agreement and negotiating a political agreement with the ELN. Finally, the article concludes that the 2018 Presidential campaign led to the polarization and political radicalization in the internal conflict resolution.

Keywords: Presidential elections; presidential speeches; Colombia; La Habana Peace Agreement; FARC-EP; ELN.

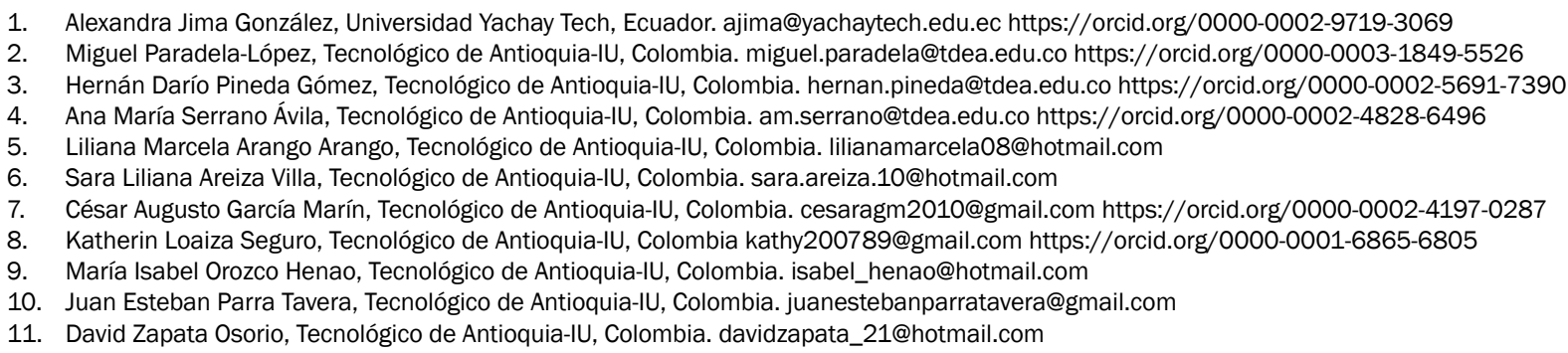

11. David Zapata Osorio, Tecnológico de Antioquia-IU, Colombia. davidzapata_21@hotmail.com 


\section{INTRODUCCIÓN}

Probablemente el Acuerdo de Paz firmado en 2016 en La Habana entre el gobierno colombiano y las Fuerzas Armadas Revolucionarias de Colombia-Ejército Popular (FARC-EP) sea uno de los hechos más relevantes de la historia reciente del país. A través de este acuerdo finalizó un conflicto con una duración de más de cincuenta años, que había causado cientos de miles de muertes y millones de desplazados entre la población colombiana. Del mismo modo, la dinámica de superación de la confrontación que se generó con la desmovilización de las FARC-EP y su reinserción en la vida civil ha llevado a que otras organizaciones -especialmente el Ejército de Liberación Nacional (ELN)- busquen negociar tratados de desmovilización con el Estado colombiano.

No obstante, este Acuerdo de Paz se ha constituido en uno de los elementos de mayor fricción en el país, puesto que ha contado con intensos posicionamientos, tanto a favor como en contra (Ruano y Muñoz, 2019). En este sentido, ha supuesto un punto de ruptura en la población y en los principales partidos políticos, especialmente en las partes concernientes a la justicia transicional (justicia especial para los guerrilleros desmovilizados) y a la participación política (integración política de las FARC-EP a través del partido Fuerza Alternativa Revolucionaria del Común). Esta división de la sociedad quedó constatada en las elecciones a la Presidencia de la República de 2018, donde la segunda vuelta enfrentó a Iván Duque, un político marcadamente conservador, con Gustavo Petro, antiguo integrante de la guerrilla urbana M-19.

En tal coyuntura, el objetivo de este artículo es realizar un análisis de los candidatos a la presidencia de Colombia -o de candidatos que comenzaron la carrera electoral a la presidencia, pero posteriormente renunciaron- para estudiar cómo el resultado de la elección afectará a la superación de la confrontación armada del país. Más concretamente se han escogido las candidaturas de Gustavo Petro, Sergio Fajardo, Humberto De la Calle, Germán Vargas Lleras, Iván Duque, Piedad Córdoba, Viviane Morales, Juan Carlos Pinzón y Rodrigo Londoño.

Para poder realizar esta aproximación se procede a examinar tres elementos de cada candidato: sus propuestas programáticas -tanto del candidato como de los partidos o alianzas que lo componen-, sus comparecencias públicas -en entrevistas o ruedas de prensa- y sus comunicaciones a través de las redes sociales -esencialmente a través de las páginas oficiales y las cuentas de Twitter y Facebook.

\section{METODOLOGÍA}

El presente artículo se desarrolla a través de un análisis exploratorio cualitativo de las campañas electorales en la carrera presidencial con respecto a la negociación con los grupos guerrilleros. Para ello, se construyó una conceptualización histórica para entender la coyuntura y la importancia de las elecciones presidenciales en Colombia, haciendo hincapié en el proceso de superación de la violencia armada que atraviesa el país después de la firma del Acuerdo de Paz con las FARC-EP en 2016 y el desarrollo de negociaciones para la desmovilización con el ELN. En este sentido, la presente investigación complementa, a través de un análisis cualitativo, el estudio cuantitativo llevado a cabo por el Semillero en Geopolítica del Tecnológico de Antioquia en 2018 que analizó los elementos que influyeron en el voto al Acuerdo de Paz de La Habana (Jima et al. 2018).

Para delimitar los casos de estudio, se procedió a escoger la muestra de candidatos a la presidencia de Colombia con base en los resultados electorales de la primera y segunda vuelta electoral (véase Tabla 1), que dio lugar a la victoria del candidato de Centro Democrático, Iván Duque, frente al representante de Colombia Humana, Gustavo Petro.

Tabla 1. Resultados elecciones presidenciales ( $1^{a}$ y $2^{a}$ vuelta)

\begin{tabular}{|c|c|c|}
\hline Candidato & Porcentaje de voto en primera vuelta electoral & Porcentaje de voto en segunda vuelta electoral \\
\hline Iván Duque & $39.4 \%$ & $54.03 \%$ \\
\hline Gustavo Petro & $25.1 \%$ & $41.77 \%$ \\
\hline Sergio Fajardo & $23.8 \%$ & \\
\hline Germán Vargas Lleras & $7.3 \%$ & \\
\hline Humberto De la Calle & $2 \%$ & \\
\hline
\end{tabular}

Fuente: Elaboración propia a partir de MOE (2018). 
En este sentido, el estudio se ha centrado en los candidatos Iván Duque (Centro Democrático), Gustavo Petro (Coalición Petro Presidente), Sergio Fajardo (Coalición Colombia), Germán Vargas Lleras (Coalición Mejor Vargas Lleras) y Humberto De la Calle (Coalición Partido Liberal y Partido Alianza Social Independiente). Por tanto, se ha decidido no abordar otras candidaturas que, si bien concurrieron a las elecciones, obtuvieron menor apoyo o directamente retiraron su candidatura, como son Piedad Esneda Córdoba Ruiz, Viviane Aleyda Morales Hoyos, Juan Carlos Pinzón Bueno o Rodrigo Londoño Echeverri (Timochenko).

Para estudiar la línea programática de los candidatos, se procedió a revisar sus programas de gobierno oficiales, sus apariciones públicas y a analizar sus manifestaciones a través de las redes sociales (véase Tabla 2). Más concretamente, se analizaron las propuestas formuladas con respec- to a las negociaciones con el ELN y sus posturas oficiales con respecto al Acuerdo de Paz firmado con las FARC-EP, tanto a través de sus programas electorales como mediante las comparecencias públicas y los vídeos y mensajes de los candidatos en sus redes sociales.

\section{CONCEPTUALIZACIÓN HISTÓRICA}

El nacimiento del bipartidismo liberal-conservador se configura a principios del siglo XIX, durante el proceso de ruptura con el orden colonial y el surgimiento del periodo republicano, configurado por una serie de tensiones entre vertientes de la élite que pugnaban por la ruptura con los valores de la monarquía española, frente a otros grupos neogranadinos que buscaban su conservación, conflicto que fue delimitando la base ideológica de los futu-

Tabla 2. Resultados elecciones presidenciales ( $1^{a}$ y $2^{a}$ vuelta) Ejes programáticos y discursivos de los principales candidatos a las elecciones presidenciales

\begin{tabular}{|c|c|c|}
\hline Candidato & Eje Programático & Eje discursivo \\
\hline $\begin{array}{l}\text { Iván } \\
\text { Duque }\end{array}$ & $\begin{array}{l}12 \text { Propuestas para seguridad. } \\
\text { Legalidad Emprendimiento Equidad }\end{array}$ & $\begin{array}{c}\text { Página web: } \underline{\text { https://www.ivanduque.com/\#openModal }} \\
\frac{\text { http://www.centrodemocratico.com/ }}{\text { Redes sociales: ivanduquemarquez }} \\
\text { @IvanDuque }\end{array}$ \\
\hline $\begin{array}{l}\text { Gustavo } \\
\text { Petro }\end{array}$ & Colombia Humana hacia una era de paz & $\begin{array}{l}\text { Página web: } \text { https://petro.com.co/ } \\
\text { http://www.progresistascolombia.org/ } \\
\text { Redes sociales: GustavoPetroUrrego } \\
\text { @petrogustavo }\end{array}$ \\
\hline $\begin{array}{l}\text { Sergio } \\
\text { Fajardo }\end{array}$ & $\begin{array}{c}\text { Bases programáticas de la Coalición Colombia } \\
\text { Partido Alianza Verde, Polo Democrático } \\
\text { Alternativo y Compromiso Ciudadano }\end{array}$ & $\begin{array}{l}\text { Página web: } \underline{\text { http://sergiofajardo.co/ }} \\
\text { https://www.claudia-lopez.com/ } \\
\text { Redes sociales: AquiEnColombia } \\
\text { @compromisociu } \\
\text { @sergio_fajardo }\end{array}$ \\
\hline $\begin{array}{l}\text { Germán } \\
\text { Vargas } \\
\text { Lleras }\end{array}$ & Nuestra Política Pública de Justicia & $\begin{array}{c}\text { Página web: https://www.mejorvargaslleras.com/ } \\
\begin{array}{c}\text { http://www.partidocambioradical.org/ } \\
\text { http://www.juancarlospinzon.co/ }\end{array} \\
\text { Redes sociales: VargasLleras2018 } \\
@ \text { German_Vargas }\end{array}$ \\
\hline $\begin{array}{l}\text { Humberto } \\
\text { De la Calle }\end{array}$ & La Paz en Marcha & $\begin{array}{c}\text { Página web: } \text { https://www.humbertodelacalle.co/ } \\
\text { https://www.partidoliberal.org.co/ } \\
\text { Redes sociales: DeLaCalleHum } \\
\text { @DeLaCalleHum }\end{array}$ \\
\hline
\end{tabular}

Fuente: Elaboración propia. 
ros partidos liberal y conservador (Moreno, García y Clavijo, 2010; Ruiz, 1997). De acuerdo con Moreno, García y Clavijo (2010), el periodo de construcción de esa disputa ideológica se remonta a los años 30 y 40 del siglo XIX, cuando se marca la ruptura con las ideas de Bolívar y la desintegración del proyecto de Santander entre liberales moderados y rojos. No obstante, aunque durante el siglo XIX se presentan tensiones con respecto a los temas de la religión y el acceso a la tierra, existía un consenso en temas económicos entre sectores de ambos movimientos (Ruiz, 1997) y una clara tendencia a la exclusión de otras fuerzas y expresiones.

Durante el siglo XIX se desarrollaron nueve guerras civiles como resultado de la pugna entre liberales y conservadores, así como numerosos conflictos regionales y locales (Tirado Mejía, 1996). De acuerdo con Ruiz (1997), un factor que aporta a explicar este fenómeno es qué ningún partido en el transcurso del siglo XIX e inicios del siglo XX fue lo suficientemente hegemónico para materializar sus idearios. En este contexto, el primer acuerdo de paz oficial entre liberales y conservadores fue firmado en 1902 para poner fin a la Guerra de los Mil Días. Acabado este conflicto, inicia una hegemonía de gobiernos de corte conservador que durará 30 años (Posada, 2002). De 1930 a 1946, se reconfiguran las fuerzas y suben al poder los liberales, quienes centraron sus esfuerzos en la institucionalización y la solución del conflicto agrario del país. Del mismo modo, buscaron apoyar y nutrirse de los grupos sindicales y profundizar la separación entre la Iglesia Católica y el Estado. En 1946 sube al poder el conservador Mariano Ospina Pérez, inicián- dose un nuevo ciclo de violencia bipartidista, conocida como la época de "La Violencia", que dejó más de 300.000 muertes.

Este periodo termina en 1953, con el golpe de Estado de Rojas Pinilla y en 1957 con el pacto oficial entre liberales y conservadores. Este tratado estableció el acuerdo denominado como Frente Nacional, pacto firmado entre los dos partidos para gobernar alternadamente durante dieciséis años. No obstante, este periodo dio lugar al surgimiento de varios grupos insurgentes, entre ellos las desmovilizadas FARC-EP, el Ejército de Liberación $\mathrm{Na}$ cional (ELN) y el Ejercito Popular para la Liberación (EPL) (Ugalde \& Freytter, 2014).

Una vez terminado el pacto del Frente Nacional se establece una serie de políticas con el objetivo de acabar con el conflicto social existente, que continuaba teniendo en el centro de la problemática el acceso a la tierra y la lucha por el poder. Estas políticas fueron acusadas de estar únicamente encaminadas a lograr la Desmovilización, Desarme y Reinserción (DDR) de los grupos insurgentes, sin proponer verdaderos cambios estructurales que respondiesen a las principales problemáticas de la sociedad.

La siguiente figura resume los acuerdos de paz oficializados por los diferentes gobiernos presidenciales desde 1984 hasta la actualidad.

Del mismo modo, a continuación, la Tabla 3 presenta algunas de las acciones encaminadas a solucionar el conflicto en Colombia y desarrolladas durante los diferentes gobiernos presidenciales desde 1978.

Figura 1. Acuerdo de Paz 1984-2016

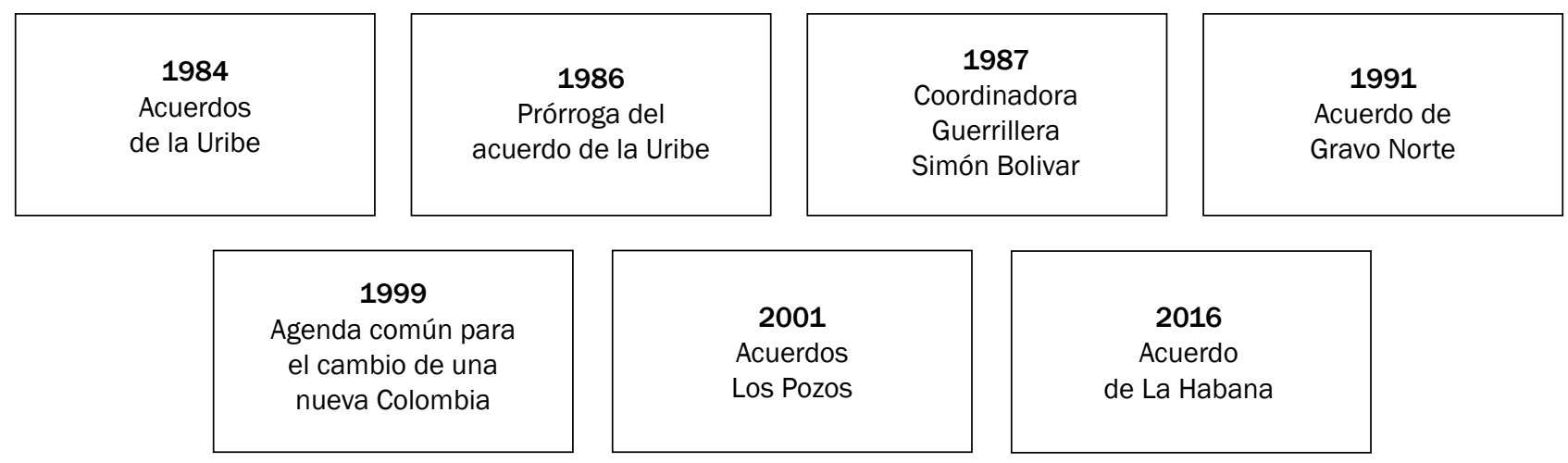


Tabla 3. Presidentes y acciones hacia la paz

\begin{tabular}{|c|c|c|}
\hline $\begin{array}{c}\text { Presidente } \\
\text { Año de gobierno }\end{array}$ & Partido & Acciones \\
\hline $\begin{array}{l}\text { Julio César } \\
\text { Turbay Ayala } \\
1978-1982\end{array}$ & Liberal & - Se desarrolla el Estatuto de Seguridad Nacional y se estable un Comité de Paz. \\
\hline $\begin{array}{l}\text { Belisario } \\
\text { Betancur } \\
1982-1986\end{array}$ & Conservador & $\begin{array}{l}\text { - Se crea la Comisión Nacional para negociar con el M19, el EPL y el ADO. } \\
\text { - Se crean comisiones de diálogos y verificación. } \\
\text { - Se logra acuerdos con dos destacamentos del ELN y se prorrogan los acuerdos } \\
\text { con las FARC-EP. }\end{array}$ \\
\hline $\begin{array}{l}\text { Virgilio } \\
\text { Barco } \\
1986-1990\end{array}$ & Liberal & $\begin{array}{l}\text { - Persecución y asesinato de dirigentes políticos de la UP. } \\
\text { - Se crea la Consejería para la Reconciliación, Normalización y Rehabilitación. }\end{array}$ \\
\hline $\begin{array}{l}\text { Cesar } \\
\text { Gaviria Trujillo } \\
1990-1994\end{array}$ & Liberal & $\begin{array}{l}\text { - En } 1991 \text { se logran acuerdos de DDR con: EPL, PRT y Quintin Lame. } \\
\text { - Se re-estructura la Consejería para la Paz. } \\
\text { - En } 1993 \text { se consigue DDR: La corriente de Renovación Socialista, las CAP } \\
\text { y con uno de los frentes del EPL. }\end{array}$ \\
\hline $\begin{array}{l}\text { Ernesto } \\
\text { Samper } \\
1994-1998\end{array}$ & Liberal & $\begin{array}{l}\text { - Ley } 368 \text { de } 1997 \text { por medio de la cual se busca financiar las acciones de paz. } \\
\text { - Logra DDR con un pequeño grupo del M-19. } \\
\text { - } 1998 \text { con la Declaración de Mainz, se crea un comité para una posible } \\
\text { convención con el ELN. }\end{array}$ \\
\hline $\begin{array}{l}\text { Andrés } \\
\text { Pastrana } \\
1998-2002\end{array}$ & Conservador & $\begin{array}{l}\text { - Se inician conversaciones con las FARC-EP y logran protocolizar } 12 \text { puntos de la } \\
\text { Agenda Común por el cambio. Se crea la zona de distención para los diálogos. } \\
\text { - Se crea comisión de países facilitadores, Canadá, Cuba, España, Francia, Suecia } \\
\text { entre otros. }\end{array}$ \\
\hline $\begin{array}{c}\text { Álvaro } \\
\text { Uribe Vélez } \\
\text { 2002-2010 }\end{array}$ & $\begin{array}{l}\text { Partido } \\
\text { de la U }\end{array}$ & $\begin{array}{l}\text { - Se logra DDR con el grupo contrainsurgente Autodefensas Unidas de Colombia AUC. } \\
\text { - Se emite la ley } 975 \text { de } 2005 \text {. Ley de justicia y paz. }\end{array}$ \\
\hline $\begin{array}{l}\text { Juan Manuel } \\
\text { Santos } \\
2010-2018\end{array}$ & $\begin{array}{l}\text { Partido } \\
\text { de la U }\end{array}$ & $\begin{array}{l}\text { - Se firma el acuerdo de paz con el grupo insurgente FARC-EP. } \\
\text { - Se crea una mesa de dialogo con ELN. }\end{array}$ \\
\hline
\end{tabular}

Fuente: Elaboración propia a partir de Ugalde y Freytter (2014).

A partir del siglo XXI, el nacimiento de nuevos partidos y movimientos políticos, tales como la Alianza Social Independiente (ASI), el Movimiento Independiente de Renovación Absoluta MIRA (2000), el Polo Democrático Alternativo (2006), el Partido Alianza Verde (2005), el Partido de la U (2005), el Partido Cambio Radical (2005) y el Centro Democrático (2013), evidencia la superación del sistema de partidos tradicional y la llegada de gobiernos alternativos.

\section{1. Élite y poder político en Colombia}

Esta línea de acontecimientos históricos en el siglo XX se desarrolla en un marco de tensión/alianza entre élites tradicionales, con tendencias faccionalistas y, personalistas (Ruiz, 1997) al mismo tiempo que prácticas de caciquismo y líderes locales (Martín, 2018). A través de este proceso se configura un sistema tradicionalista/clientelista, con presencia de proyectos regionales que alternativamente conviven o se enfrentan a los gobiernos de las élites centrales.

De acuerdo con Ruiz (1997), el periodo de mayor tensión se da entre "la constitución de 1886 como un proyecto político de los terratenientes, chocara con el que querían construir los comerciantes, marcado por el liberalismo económico, la secularización y el trabajo libre remunerado salarialmente" (p. 79). No obstante, este conflicto posteriormente se unió con el proceso de modernización capitalista de inicios del siglo XX, dónde "consolidados como 
élites socioeconómicas, las diferencias de los partidos perdían relevancia y las posibilidades de coalición y gobiernos compartidos se afianzaba cada vez más" (p. 80). En este sentido, Martín (2018) habla de un "nacionalismo modernizador" (p. 28), refiriéndose al proyecto que aglutinó facciones de la élite bajo un modelo agroexportador y librecambista. Este movimiento "sirvió para convencer a las capas sociales medias y bajas -en especial, a los artesanos, que abogaban por un mayor proteccionismo estatal -de que se lograría un mayor progreso económico y social y que los ciudadanos serían libres e iguales" (p. 28).

Más concretamente, Martín (2018) distingue tres etapas en la conformación de las élites tradicionales: 1) Las décadas anteriores de la época de La Violencia y la creación del Frente Nacional; b) Los años de desarrollo del Frente Nacional; y 3) el periodo posterior al Frente Nacional. Cómo elemento trasversal se encuentran los elementos de aglutinación y disputa con élites locales, así como los procesos de cerramiento o límites a la democracia. En este sentido, durante el siglo XX se van a materializar diversos tipos de pactos de élites, incluyendo diferentes sectores políticos con el propósito de mantener el control político del país. Como resultado, "las élites dominantes han colocado al Estado al servicio de sus intereses políticos y económicos, involucrándose en una lucha por el poder siendo los partidos políticos los celosos guardianes del modelo excluyente" (Ruiz, 1997, p. 87), factor de una violencia estructural (Moreno, 2018).

\section{ANÁLISIS DE LOS CANDIDATOS}

\subsection{Gustavo Petro}

Desde un punto de vista político, la candidatura de Gustavo Petro puede considerarse como la que más polémica ha desatado, ya que sufrió diversas acusaciones en torno a su carácter radical. En este sentido, ha sido frecuentemente acusado por políticos, tanto de la esfera progresista como conservadora, de querer implementar un régimen político similar al de Cuba y Venezuela. Del mismo modo, debido a su pasado como antiguo guerrillero del M-19, se le ha acusado de estar aliado con el ahora partido Fuerza Alternativa Revolucionaria del Común (anteriormente la guerrilla FARC-EP) (Portela 2017).

No obstante, la ventajosa posición en la que se encontró -encabezando los sondeos junto con el candidato del Centro Democrático Iván Duque- evidencia cómo el nicho de votos que apoya a Petro se extiende mucho más que el situado en la izquierda radical, aglutinando parte del voto anti-establishment que se opone a la vieja clase política colombiana.

Con respecto a la postura política sobre la superación del conflicto armado de Colombia, destaca en un primer momento la ausencia de un cuerpo programático centrado en el mismo, tanto en lo que se refiere a la figura de Petro como a la de su proyecto Colombia Humana. Esta aparente ausencia puede explicarse a través de dos elementos: las acusaciones de colaboracionismo con la guerrilla y una concepción de la resolución de la guerra en un sentido amplio.

En referencia a la primera cuestión, cabe suponer que la asociación con el antiguo movimiento guerrillero y con el ELN haya provocado que la temática de la finalización del conflicto de Colombia fuese relegada a un segundo plano, en virtud de otra serie de cuestiones sociales. Con respecto a la segunda cuestión, Petro ha defendido la necesidad de acudir no únicamente a la solución del conflicto con las FARC-EP y con el ELN, sino también de actuar sobre los problemas sociales que padece la sociedad colombiana y que, según él, generaron el surgimiento de los movimientos guerrilleros.

Esta concepción puede observarse en el título del programa político de Gustavo Petro: "Colombia Humana hacia una era de paz". Según su argumentario, la pacificación de Colombia no puede conseguirse únicamente a través de acuerdos de paz con los movimientos guerrilleros, sino que son necesarias políticas generales sobre las siguientes temáticas: salud, educación, economía, política y justicia. Según manifiesta en su cuenta oficial a través de la plataforma Facebook: "La paz no es el final de una guerra. La paz es una serie de transformaciones reales de la sociedad" (Petro, 2018).

Por otro lado, resulta de especial interés un giro retórico que Petro ha utilizado a la hora de defenderse de la acusación de querer convertir a Colombia en una nueva Venezuela. Desde su cuenta de Facebook, Petro ha manifestado en reiteradas ocasiones que Colombia ya se ha convertido en una nueva Venezuela, a causa de la corrupción y la ineficiencia de los partidos políticos clásicos: “El grado de inteligencia de Santos, Uribe, Pastrana y Lleras es el mismo de Maduro, ellos han convertido a Colombia en Venezuela" (Petro 2018). Por tanto, Petro señala a las prácticas de clientelismo, belicismo y corrupción como los grandes impedimentos de la consolidación de la paz en Colombia. Y, por tanto, responsabiliza a los diri- 
gentes de los antiguos gobiernos de Uribe y Santos como los grandes estructuradores de la "derecha anti-Paz" que frenan el fin de la guerra en Colombia.

Sobre este apartado cabe resaltar una cercanía entre los postulados sostenidos por Petro y lo establecido en el Acuerdo de Paz de 2016, destacando el plan de desarrollo agrario o la mejora en la distribución de la riqueza. Estas similitudes invitan a considerar que Petro, desde un punto de vista programático, tenderá a mantener el cumplimiento de Acuerdo de Paz sin realizar cambios significativos sobre el mismo. La propia denominación de la consulta que tuvo lugar el 11 de Marzo entre los candidatos Petro y Caicedo, Ilamada Inclusión Social por la Paz, evidencia el compromiso de esa fuerza por la pacificación del país.

Del mismo modo, dado su compromiso con la construcción de la paz en Colombia, cabe esperar una actitud favorable a la negociación de un alto el fuego definitivo con el ELN. Esta actitud favorable ya se pudo observar en su posición con respecto a la tregua unilateral y al inicio de negociaciones entre el Estado y el ELN. En este sentido ha afirmado a través de sus redes sociales que el final de la guerra sería un éxito histórico para el país (Petro 2018).

En definitiva, Petro puede definirse como un candidato de marcado carácter progresista que señala la consolidación de la paz en Colombia como una de las prioridades de su gobierno. Con respecto al Acuerdo de Paz y las negociaciones con el ELN, se ha manifestado de acuerdo con ambas, argumentando la necesidad del fin de los conflictos guerrilleros para el comienzo de la construcción de una sociedad más justa. De hecho, se ha defendido que el pasado de Petro en el M19 podría facilitar la negociación y el acuerdo con los dirigentes del grupo guerrillero, ya que el político jugó un papel clave en la propia desmovilización de su antigua organización revolucionaria (El Colombiano, 2018).

\subsection{Sergio Fajardo}

En 2016, Fajardo hizo campaña a favor de los acuerdos con las FARC-EP en el Plebiscito por la Paz. De hecho, la coalición de su candidatura presidencial ha expresado pública y reiteradamente que apoya los acuerdos de paz, afirmando que el único camino real para resolver el conflicto es el diálogo. En entrevista con Dussan (Semana en vivo, 2017), Fajardo afirmó estar a favor del acuerdo de paz, aunque expuso que van a recibir un proceso maltrecho debido a que la pedagogía para la paz no se hizo desde el comienzo: no se explicaron las razones de la paz, lo que hizo más difícil comprender la importancia de alcanzar el acuerdo. Acudiendo al programa de la Coalición Colombia: "Las guerras nacen en la mente de los hombres, y es en la mente de los hombres donde deben erigirse los baluartes de la paz" (Coalición Colombia 2018: 26).

En todo caso, Fajardo afirmó que "Nosotros tenemos que desarrollar lo que nos entreguen", mostrando su respaldo hacia el Acuerdo de Paz firmado en La Habana: "Por convicción esta coalición asume el compromiso de consolidar la paz territorial y defender e implementar los Acuerdos de Paz suscritos y otros que se puedan lograr" (Coalición Colombia 2018).

Con respecto a los acuerdos de paz con el grupo insurgente ELN, en una entrevista realizada por Caracol Radio, Fajardo expresó que este grupo había perdido muchas oportunidades para iniciar un proceso con el gobierno colombiano. Sin embargo, resaltó la urgente necesidad de iniciar un proceso de desmovilización y cese al fuego.

Adicionalmente, en medio de la campaña presidencial, Fajardo alentó a la fuerza pública a mantener toda ofensiva contra esta guerrilla y a no bajar la guardia. Defendió que la decisión del grupo insurgente de retomar los actos violentos perpetuados en diferentes partes del país evidenciaba la voluntad de abandonar la vía de la solución pacífica del conflicto. En entrevista en El Colombiano, manifestó la necesidad de conformar un equipo negociador por parte del gobierno (El Colombiano, 2018).

\subsection{Humberto De la Calle}

Para las elecciones presidenciales de 2018, De la Calle se postuló como candidato por el Partido Liberal. Bajo el título de La Paz en Marcha, su programa político señala como objetivo principal consolidar la paz que Colombia comenzó a construir con el Acuerdo de paz (De la Calle 2018). Por tanto, el mantenimiento del acuerdo entre el Estado colombiano y las FARC-EP se convirtió en una de sus principales prioridades, tal y como puede observarse en una entrevista realizada en el año 2017:

(...) Ias FARC entregaron las armas, han entregado el inventario de sus bienes, se concentraron en las zonas veredales. Yo creo que en la práctica ambas partes están cumpliendo lo sustancial. Ahora, para mí lo que afronta Colombia no es tanto la discusión sobre el acuerdo, sino sobre las oportunidades que brinda el haber terminado un conflicto. (Arias Herrera, 2017, párr. 1) 
En este sentido, la campaña de De la Calle se apoya en un esquema político donde el posconflicto es clave. Más concretamente, afirma su apoyo a lo pactado en La Habana, aunque sí manifiesta su voluntad de realizar cambios menores en algunos puntos de índole económico. De la Calle incluso manifestó que uno de sus "temores" en la campaña electoral era el incumplimiento del Acuerdo de Paz, ya que causaría una vuelta a nuevas formas de violencia (Arias Herrera, 2017). En definitiva, De la Calle propone una candidatura a la presidencia enfocada en continuar con el proceso de consolidación de la paz, sustentada tanto en el mantenimiento del acuerdo con las FARC-EP como en la educación, realizando una labor pedagógica que extendiese la cultura pacifista al ámbito político, económico y social.

Con respecto al posible diálogo con el ELN, se ha mostrado abierto a la negociación con otros movimientos guerrilleros de Colombia con el propósito de extender el proceso de pacificación a zonas históricamente conflictivas. A principios del 2016, en una entrevista realizada por periodistas de RCN, De la Calle se dirigió al ELN para invitarles a ser parte de la superación del conflicto armado y así comenzasen su proceso de desmovilización y reinserción en la vida civil (Noticias RCN, 2016). En este mismo pronunciamiento De la Calle agregó:

No importa en qué orilla nos encontremos, todos tenemos que dejar claro que, por encima de la discusión democrática, existe un propósito común en la defensa de la unidad de los colombianos y en pro de los valores esenciales que son necesarios para la convivencia como sociedad. (Noticias RCN, 2018, párr. 4)

Por tanto, la postura de De la Calle frente al ELN está centrada en mantener conversaciones con el grupo guerrillero de cara a alcanzar un proceso análogo al logrado con las FARC-EP. De hecho, tras los actos de violencia perpetuados por el ELN en el transcurso de las conversaciones con este grupo a inicios del 2018, el candidato expresó su rechazo por lo sucedido, alentando a retomar el diálogo y la consecución de un acuerdo de paz. Uno de los hechos más sonados de su campaña fue el llamamiento de De la Calle al resto de los candidatos a la presidencia de Colombia para generar una suerte de consenso en la construcción de un diálogo que garantice la negociación y el fin del conflicto con el ELN. En definitiva, es un candidato que apuesta fuertemente por el mantenimiento del Acuerdo de Paz con las FARC-EP y por el establecimiento de negociaciones con el ELN dirigidas a alcanzar el fin del conflicto armado.

\subsection{Germán Vargas Lleras}

La postura de este político con respecto al Acuerdo de Paz firmado en La Habana no es del todo clara, ya que no están recogida dentro de sus propuestas de campaña. En este sentido, la presente sección se basa en las declaraciones que ha hecho mediante entrevistas en medios de comunicación. En ellas puede observarse que muestra un discurso inconstante, ya que oscila entre el apoyo al Acuerdo de Paz con fuertes críticas y propuestas de renovación del mismo.

Así, en la entrevista concedida a la Revista Semana (2016), y previo al plebiscito de octubre del 2016, Vargas Lleras dio su primer comentario sobre los acuerdos, manteniendo que apoyaría el Sí, aunque mostrando cierto rechazo a algunas partes del Acuerdo por considerarlas demasiado beneficiosas para la guerrilla. En diálogo con Noticias Caracol (2017), afirmó lo siguiente: “(...) soy el colombiano más feliz con que esta organización se haya desmovilizado. La entrega de las armas me parece extraordinaria. La posibilidad de que puedan hacer política es una aspiración lógica del acuerdo".

Posteriormente parece endurecer su postura, ya que en conversación con el Diario Proclama del Cauca (2018) sostuvo la necesidad de revisar el acuerdo. En este sentido, expresó críticas a la Justicia Especial de Paz, que según él liberaba de responsabilidad por graves delitos a los desmovilizados. Del mismo modo, expresó duras críticas hacia el programa de destrucción de los cultivos ilícitos, ya que considera que no había tenido éxito. En definitiva, parece evidenciarse que Vargas Lleras mantiene una posición de respetar la estructura del acuerdo, aunque realizando modificaciones de peso. Sin embargo, al mismo tiempo ha desarrollado una postura en la que reconoce la necesidad de integrar a las FARC-EP en la vida política nacional.

Al igual que con el Acuerdo de Paz de La Habana, el candidato no ha manifestado una postura oficial en su sitio web de campaña con respecto a las negociaciones de paz con el ELN. Sin embargo, sí ha manifestado sus opiniones en entrevistas, en las que ha mostrado una postura más directa y contraria al diálogo, llegando a afirmar que si fuese presidente no retomaría las negociaciones de paz con el ELN, al considerarlo un intento de "doblegar a Colombia" (El Espectador, 2018).

En la Revista Semana (2018), comparó la negociación con el ELN con las conversaciones de paz del Caguán (Caquetá) de 1998. Mediante esta comparación, Vargas Lleras dio a entender que este posible acuerdo sería negociado con una intención 
del ELN de aumentar su capacidad militar. Por tanto, respecto a una posible negociación con el ELN, Vargas Lleras muestra una postura escéptica:

Me da la impresión de que este proceso comenzó mal y no va a conducir a puerto seguro, porque si el piso con el cual iniciaron las negociaciones fueron los acuerdos de La Habana, imagínense cuáles serán las pretensiones adicionales que querrán. Hay que acabar estos diálogos. (párr. 9)

En conclusión, se podría decir que Vargas ha hecho una serie de observaciones que permiten vislumbrar cambios al Acuerdo de Paz con las FARC$\mathrm{EP}$, así como su voluntad de dar por terminadas las negociaciones de paz con el ELN, sustituyéndolas por una campaña militar y policial que obligue a la organización guerrillera a rendirse.

\subsection{Iván Duque}

En cuanto a los acuerdos de La Habana, en entrevista realizada por la Revista Semana en 2017, afirmó que no está en desacuerdo con la construcción de un proceso negociado, siempre y cuando la negociación sea producto del triunfo de la legalidad y del Estado de Derecho. Duque considera que el acuerdo firmado en La Habana ha dado graves concesiones que ponen a la criminalidad por encima del Estado de Derecho, debido a que se les abre la posibilidad de participación política a los máximos líderes, quienes en su opinión deberían responder por crímenes de Lesa Humanidad (Semana, 2017).

En este sentido, sostiene la idea de revisar algunas partes de los acuerdos de paz con las FARC-EP, sobre todo en el punto segundo, centrado en la participación política. Adicionalmente, afirmó que no se opone a que se aplique la ley de amnistía a la base del grupo insurgente y está de acuerdo con desarrollar programas sociales para la reincorporación e inserción de los desmovilizados. Sin embargo, se opone a que los cabecillas de las FARC-EP participen en política, ya que cree que los crímenes cometieron deben ser castigados.

Por otro lado, en una entrevista para CNN (Egaña, 2018), expresó estar en acuerdo con una desmovilización, desarme y reinserción del grupo insurgente Ejército de Liberación Nacional - ELN. Sin embargo, mencionó que este proceso debía darse en un contexto de garantías y condiciones, entre ellas la concentración previa con supervisión internacional, la suspensión de todas las actividades criminales, el establecimiento de un plazo para su desmovilización y la reducción de penas sin amnistía.
Por otro lado, en su programa político Duque resalta la importancia de enfrentar militarmente a las fuerzas guerrilleras, en las que incluye al mismo nivel al ELN, al narcotráfico, a las bacrims y a las disidencias de las FARC-EP. Del mismo modo, propone "prohibir en la Constitución la admisibilidad del narcotráfico como delito político conexo. El narcotráfico no podrá ser un delito amnistiable en Colombia" (Duque, 2018, p. 4). Esta disposición invita a considerar la posibilidad de que su llegada a la presidencia no sólo dificultaría la negociación con el ELN, sino que también supondría la revisión del acuerdo con las FARC-EP.

Adicionalmente en entrevista realizada en enero del 2018 por un periódico local en Bucaramanga argumentó que en su gobierno se perseguirán a los grupos criminales con toda la capacidad de la fuerza pública, incluyendo al ELN. Por tanto, el candidato Iván Duque es probablemente aquel que tiene una postura más beligerante hacia el proceso de finalización del conflicto, puesto que plantea una revisión en profundidad del Acuerdo de Paz con las FARC-EP y un margen de negociación con el ELN casi inexistente.

\section{CONCLUSIONES}

Del presente estudio puede afirmarse cómo la campaña electoral de 2018 reflejó las discusiones derivadas de los Acuerdos de La Habana de 2016. Así, mientras para ciertos candidatos el Acuerdo de Paz significaba el cierre de un capítulo de la historia del país, para otros implicaba la derrota ante los movimientos insurgente. Estos elementos discursivos se vieron reflejados en los programas políticos y en la actividad de las redes sociales de los principales candidatos que compitieron a la Presidencia de la República.

Con respecto al mantenimiento de los Acuerdos de La Habana, pueden distinguirse dos grupos de posturas: uno dirigido a rectificar, ajustar o modificar el acuerdo suscrito (Iván Duque y Germán Vargas), y otro centrado en el respeto y cumplimiento del contenido del acuerdo (Sergio Fajardo, Gustavo Petro y Humberto De la Calle). En relación con el ELN, existen ciertas diferencias, ya que, al tratarse de un conflicto vigente durante la campaña electoral, todos los candidatos compartieron la exigencia de que el grupo guerrillero abandonase la vía militar. No obstante, pueden distinguirse dos grupos, dependiendo de si abogaron por el man- 
tenimiento de mesas de diálogo destinadas a una salida negociada o si defendieron la presión armada para provocar su disolución. Esta división tan marcada muestra cómo los discursos de la paz continúan siendo un tema relevante para la agenda política colombiana.

Por otro lado, si bien dentro del grupo de candidatos se pueden identificar las vertientes descritas, es importante reseñar que la mayoría de candidatos mostraron una falta de concreción acerca de las acciones puntuales a realizar en caso de ganar la elección presidencial. Esto tiene explicación en que la Constitución Política de Colombia y la Ley Electoral no establecen el voto programático en Colombia para las elecciones presidenciales, por lo que no resulta obligatorio registrar un programa de gobierno cuando se inscribe la candidatura.

Con el propósito de sintetizar el contenido analizado en los apartados anteriores, a continuación, se muestra una tabla que resume el posicionamiento de los candidatos a la Presidencia sobre el cumplimiento del Acuerdo de La Habana y sobre la posible negociación con el ELN.

De la anterior tabla se evidencia la fuerte polarización que experimenta la sociedad colombiana con respecto a la resolución del conflicto con las FARC-EP y el ELN. En este sentido, los candidatos con mayor apoyo electoral tienen posturas marcadamente antagónicas: mientras que Iván Duque, el candidato de Centro Democrático que obtuvo la victoria, mostró un rechazo frontal a la negociación con el ELN y propuso revisar en profundidad el Acuerdo de Paz con las FARC-EP; Gustavo Petro, candidato con el $41 \%$ de los votos de los colombianos, defendió el mantenimiento de la negociación con el ELN y el respeto de los Acuerdos de La Habana en su actual extensión.

Por tanto, puede concluirse que las elecciones presidencias de 2018 han implicado un aumento de la polarización política en la que los dos candidatos que disputaron la Presidencia de la República propusieron versiones antagónicas de cómo debe resolver uno de los conflictos contemporáneos más extensos en duración y en cantidad de víctimas. 5.

Tabla 4. Síntesis de las posturas de los aspirantes en relación con los procesos de negociación con las FARC-EP y el ELN

\begin{tabular}{|c|c|c|}
\hline Candidatos & Postura con respecto a los Acuerdos de La Habana & Postura con respecto a la negociación con el ELN \\
\hline $\begin{array}{l}\text { Iván } \\
\text { Duque }\end{array}$ & $\begin{array}{l}\text { Plantea modificar los Acuerdos de La Habana para que } \\
\text { el narcotráfico no sea delito político y para revocar la } \\
\text { participación política de la cúpula de la guerrilla }\end{array}$ & $\begin{array}{l}\text { Defiende la confrontación militar en busca de su } \\
\text { desmovilización y judicialización }\end{array}$ \\
\hline $\begin{array}{l}\text { Germán } \\
\text { Vargas }\end{array}$ & $\begin{array}{l}\text { Sostiene la necesidad de revisar y modificar los Acuerdo } \\
\text { de La Habana, aunque sin aclarar los puntos concretos }\end{array}$ & $\begin{array}{l}\text { Su postura se ha dirigido al rechazo a la } \\
\text { continuación de las negociaciones }\end{array}$ \\
\hline $\begin{array}{l}\text { Sergio } \\
\text { Fajardo }\end{array}$ & $\begin{array}{l}\text { Plantea implementar lo acordado en los Acuerdos de La } \\
\text { Habana }\end{array}$ & $\begin{array}{l}\text { Su posicionamiento está más cercana a exigir } \\
\text { su desmovilización que a buscar una solución } \\
\text { negociada }\end{array}$ \\
\hline $\begin{array}{l}\text { Humberto } \\
\text { de la Calle }\end{array}$ & $\begin{array}{l}\text { Defiende implementar lo acordado en los Acuerdos de } \\
\text { La Habana }\end{array}$ & $\begin{array}{l}\text { Ha invitado a este grupo a retomar la negociación } \\
\text { y de esta forma superar la confrontación con el } \\
\text { Estado colombiano }\end{array}$ \\
\hline $\begin{array}{l}\text { Gustavo } \\
\text { Petro }\end{array}$ & $\begin{array}{l}\text { Sostiene implementar lo acordado en los Acuerdos de } \\
\text { La Habana, enfatizando en la necesidad de incidir en la } \\
\text { problemática de la distribución de la tierra }\end{array}$ & $\begin{array}{l}\text { Plantea continuar con las negociaciones como } \\
\text { solución al conflicto armado }\end{array}$ \\
\hline
\end{tabular}

Fuente: Elaboración propia. 


\section{REFERENCIAS}

Arias Herrera, M. E. (2017, 17 de agosto). El cambio sin balas es más fácil: Humberto De la Calle. Publímetro. https://www.publimetro.co/co/colombia/2017/08/17/cambio-sin-balas-mas-facilhumberto-la-calle.html

De la Calle Presidente. (2017). Humberto De la Calle Biografía. Humberto Delacalle. http://www.humbertodelacalle.co/biografia

Diario Proclama del Cauca (2018, 26 de enero). "iEl acuerdo de paz hay que revisarlo!": Vargas Lleras. Diario Proclama del Cauca. https://www. proclamadelcauca.com/el-acuerdo-de-paz-hayque-revisarlo-vargas-lleras/

Duque, I. (2018). 203 Propuestas. Iván Duque y Marta Lucía Ramírez. Duque. https://www.ivanduque.com/propuestas

Egaña, C. (2018, 9 de febrero). ¿Firmaría Iván Duque un acuerdo de paz con el ELN si llega a la presidencia de Colombia? CNN. https://cnnespanol. cnn.com/video/eln-acuerdo-paz-colombia-candidato-presidencial-intvw-ivan-duque-camilo/

El Colombiano. (2018, 13 de marzo). Diálogos con Eln deben acelerarse a fondo y buscar un cese el fuego. El Colombiano. http://www.elcolombiano.com/colombia/dialogos-con-eln-debenacelerarse-a-fondo-y-buscar-un-cese-el-fuegoGM8358967

ESAP (2017). Estrategia de Capacidades para la Paz y la convivencia. Diplomado virtual Acción CaPaz. https://www.territoriosporlapaz.gov.co/ enterese-del-proceso-de-paz\#modulos

Jima-González, A., Paradela-López, M., Pineda Gómez, H. D., Areiza Villa, S. L., García Marín, C. A., Loaiza Seguro, K. V. y Parra Tavera, J. E. (2018). El Acuerdo de Paz de la Habana. Un estudio de percepción en la población del Tecnológico de Antioquia-IU. En-Contexto, 6(9), 251-272. https://ojs.tdea.edu.co/index.php/encontexto/article/view/507

Noticias Caracol. (2017, 17 de octubre). No creo que haya que hacer trizas los acuerdos de paz": Vargas Lleras. Caracol TV. https://noticias.caracoltv. com/colombia/no-creo-que-haya-que-hacer-trizas-los-acuerdos-de-paz-vargas-Ileras

Martín de la Fuente, D. (2018). Radiografía del poder en Colombia: élites y vínculos de parentesco. Cambios y continuidades desde la teoría de redes (Tesis de Maestría). Universidad de Salamanca, Instituto de Iberoamérica.

Tirado MeJía, Á. (1996). Colombia: siglo y medio de bipartidismo. In J. O. Melo (Ed.), Colombia hoy: perspectivas hacia el siglo XXI (102-185). Tercer Mundo, Bogotá.
Mejor VARgas Lleras. (2018). Vargas Lleras - Biografía. https://www.mejorvargaslleras.com/biografia

Misión de Observatorio Electoral. (2018). Resultados electorales elecciones presidenciales. Primera y segunda vuelta. MOE. https://moe.org.co/wp-content/uploads/2018/11/Resultados-Electorales-Elecciones-Presidenciales-2018_Digital.pdf

Moreno, O., García, L. y Clavijo, J. (2010). Nacimiento del bipartidismo colombiano: pasos desde la Independencia hasta mediados del siglo XIX, Estudios Políticos, 37(1), 187-205. https://dialnet. unirioja.es/servlet/articulo?codigo $=5263874$

Moreno, H.A. (2018). Colombia: entre pactos de élites y transiciones democráticas. Entramado, 14(1), 166-179. https://doi.org/10.18041/entramado.2018v14n1.27136

Noticias RCN. (2016, 29 de enero). Humberto De la Calle llama al ELN a unirse a diálogos de paz para un acuerdo completo. RCN. http://www.noticiasrcn.com/nacional-pais/humberto-calle-llama-al-eln-unirse-dialogos-paz-un-acuerdo-completo

Noticias RCN. (2018, 29 de enero). De la Calle llama a demás candidatos a de forma conjunta fijar una posición sobre ataques del ELN. RCN. http://www. noticiasrcn.com/nacional-pais/calle-llama-demas-candidatos-forma-conjunta-fijar-una-posicion-ataques-del-eln

Petro, G. [@petrogustavo]. (2018, 26 de febrero). La tregua unilateral decretada por el ELN puede representar el final de la guerra de guerrillas insurgentes en la historia del país de manera definitiva. De lograrlo será un éxito en el camino de la construcción de una gran y poderosa nación: Colombia. [Tweet]. Twitter. https://twitter.com/petrogustavo/status/968129230151221248?lang=es

Petro, G. [@petrogustavo]. (2018-2, 28 de Febrero). La paz no es el final de una guerra. La paz es una serie de transformaciones reales de la sociedad. Construir la paz en Colombia es un tema de Humanidad. Se trata de construir un pacto social que sea capaz de construir las reformas indispensables para transformar el país. [Tweet]. Twitter. https://www. facebook.com/GustavoPetroUrrego/photos/pcb.1 0155029044885771/10155029044025771/? type=3

Petro, G. [@gustavopetrourrego]. (2018-3, 7 de abril). El grado de inteligencia de Santos, Uribe, Patrana y Lleras es el mismo de Maduro, ellos han convertido a Colombia en Venezuela. (Actualización de Estado). Facebook. https://www.facebook. com/pg/GustavoPetroUrrego/posts/

Portela García, J.C. (2017). Redes y política en la vida cotidiana. Agenda Cultural Alma Máter, 239, 
12-16. https://revistas.udea.edu.co/index.php/ almamater/article/view/328149

Posada Carbó, E. (2002). Los límites del poder: Elecciones bajo la hegemonía conservadora, 18861930. Boletín Cultural y Bibliográfico, 39, 30-65.

Revista Semana. (2016, 29 de octubre). FARC y grupos cristianos llegan a puntos comunes sobre enfoque de género. Semana. http://www.semana.com/ nacion/articulo/farc-y-cristianos-llegan-a-un-acuerdo-sobre-enfoque-de-genero/502697

Revista Semana. (2016, 27 de agosto). Vamos a derrotara las FARC, pero en las urnas. Semana. http://www. semana.com/nacion/acuerdo-final-de-paz-conlas-farc-en-2016/articulo/german-vargas-hablasobre-el-proceso-de-paz-en-exclusivo/491389

Revista Semana. (2018, 30 de enero). La izquierda radical va a llegar toda en el mismo tiquete: Vargas Lleras. Semana. http://www.semana.com/nacion/ articulo/vargas-habla-de-coalicion-con-uribe-y-critica-posible-brazo-armado-de-las-farc/555413

Ruano, L. y Muñoz, L. (2019). Plebiscito por la paz en Colombia: un análisis desde las emociones en sus resultados políticos. Ámbitos. Revista Internacional de Comunicación, 44, 110-126. https:// doi.org/10.12795/Ambitos.2019.i44.07

Ruzz, J.H. (1997). Una Visión Crítica al Bipartidismo en Colombia. Convergencia, Revista de Ciencias Sociales, 14(1), 74-92. https://convergencia.uaemex.mx/article/view/9591

Ugalde, A. y Freytter, J. (Eds.) (2014). Presente y futuro de Colombia en tiempos de esperanza. Universidad del País Vasco. 